\title{
Mechanosensing and Mechanotransduction at Cell-Cell Junctions
}

\author{
Alpha S. Yap, ${ }^{1}$ Kinga Duszyc, ${ }^{1}$ and Virgile Viasnoff ${ }^{2,3}$ \\ ${ }^{1}$ Institute for Molecular Bioscience, Division of Cell Biology and Molecular Medicine, The University \\ of Queensland, St. Lucia, Brisbane 4072, Australia \\ ${ }^{2}$ Mechanobiology Institute, National University of Singapore, Singapore 117411 \\ ${ }^{3}$ CNRS, Singapore 117411 \\ Correspondence: a.yap@uq.edu.au; virgile.viasnoff@espci.fr
}

Cell adhesion systems are defined by their ability to resist detachment force. Our understanding of the biology of cell-cell adhesions has recently been transformed by the realization that many of the forces that act on those adhesions are generated by the cells that they couple together; and that force at adhesive junctions can be sensed to regulate cell behavior. Here, we consider the mechanisms responsible for applying force to cell-cell junctions and the mechanosensory pathways that detect those forces. We focus on cadherins, as these are the best-studied examples to date, but it is likely that similar principles will apply to other molecular systems that can engage with force-generators within cells and physically couple those cells together.

$\mathrm{C}^{\mathrm{el}}$ ell-cell adhesion systems couple cells together to form mechanically coherent tissues that can resist detachment forces: This has been well-appreciated for a very long time. More recently, our understanding of the relationship between mechanical force and cell-cell adhesion has grown in range and subtlety, prompted by two advances. First was the demonstration that patterns of force that are actively driven by cells (especially contractile forces) generate morphogenetically important outcomes when they are coupled to cell-cell adhesion. This is an area that has been extensively discussed in many recent reviews (e.g., see Lecuit et al. 2011; Heisenberg and Bellaiche 2013; Martin and Goldstein 2014; Lecuit and Yap 2015). Second is the grow- ing evidence that mechanical forces can elicit active cellular responses when they impinge on, and are transmitted through, cell adhesion molecules (Orr et al. 2006; Leckband and de Rooij 2014). Effectively, mechanical force constitutes a mode of biological communication that can complement better-characterized chemical and electrical modes of biological information. We will focus on this second emerging area in the present review. Our discussion will concentrate on classical cadherins, although it is likely that other cell-cell adhesion systems will prove to support mechanosensing and mechanotransduction. For this same reason, we will principally discuss studies in epithelia and endothelia. We consider the forces that cells may

Editors: Carien M. Niessen and Alpha S. Yap

Additional Perspectives on Cell-Cell Junctions available at www.cshperspectives.org

Copyright (C) 2018 Cold Spring Harbor Laboratory Press; all rights reserved; doi: 10.1101/cshperspect.a028761

Cite this article as Cold Spring Harb Perspect Biol 2018;10:a028761 
A.S. Yap et al.

sense at cell-cell junctions; ways to measure forces at junctions; the mechanisms that allow cell-cell adhesion systems to link mechanical stimuli to functional biological outcomes; and the biological processes that are elicited by forcesensing at junctions.

\section{FORCES THAT ACT ON CELL-CELL JUNCTIONS}

Cell-cell adhesion systems experience forces that originate from a range of sources. These forces have often been characterized when tissues undergo large-scale morphogenetic movements during development or tissue remodeling. However, changes in junctional force also occur during tissue homeostasis in response to events such as apoptosis (Lubkov and Bar-Sagi 2014) and cell division (Campinho et al. 2013).

\section{External Forces Exerted at the Tissue Scale}

Cell-cell adhesion systems are exposed to, and must withstand, a range of external forces that can act on tissues. These include physical trauma, skeletal muscle contraction, and flow within blood and lymphatic vessels. It was often thought that cell-cell adhesion preserved tissue integrity by passively resisting these forces. However, apparently paradoxical responses indicate that cell adhesion systems can be actively regulated in response to the application of force. One such example is the observation that tension across vascular endothelial (VE)-cadherin decreased when shear forces were applied to vascular endothelial cells (Conway et al. 2013), whereas this would have been expected to increase if VE-cadherin were acting to passively resist force.

\section{Active Cell-Cell Forces Generated within Tissues}

Here, we refer to forces that cells exert on their neighbors. This concept has emerged with the realization that nonmuscle cells actively generate force that can be transmitted to their neighbors through cell-cell junctions (FernandezGonzalez et al. 2009; Martin et al. 2009; Rauzi et al. 2010; Ratheesh et al. 2012). Such force can be generated by a number of cellular processes, of which the best understood is contractility.

\section{Cellular Contractility}

The actomyosin system is the major contractile force-generator in eukaryotic cells (Clark et al. 2007; Vicente-Manzanares et al. 2009; Heissler and Manstein 2013). Localized changes in actomyosin activity can cause local changes in force within tissues. As we discuss below, they can also mediate the response of other cells within the tissue to those changes in force. In nonmuscle cells, this contractile apparatus involves the interaction of nonmuscle myosin II (NMII) with F-actin networks whose organization can vary substantially, from sarcomere-like structures (Ebrahim et al. 2013) and bundles (Martin et al. 2009; Smutny et al. 2010) to less-organized networks (Martin et al. 2009; Wu et al. 2014). As discussed in Mège and Ishiyama (2017), actin filaments are likely to be the principal mechanism that physically couples actomyosin to cadherin adhesion complexes. This association is mediated by $\alpha$-catenin and other actin-binding proteins that can associate with cadherins ( $\mathrm{Ra}$ theesh and Yap 2012). However, proteins like synaptopodin (Kannan and Tang 2015), which can bind both NMII and cadherin, may also contribute.

A number of distinct patterns of contractility impinge on cell-cell junctions. First, pulsatile networks characterized by oscillatory cycles of condensation and turnover are found at the medial-apical surfaces of epithelial cells in morphogenetically active tissues in Drosophila (Martin et al. 2009; Rauzi et al. 2010) and also at the lateral cell-cell junctions in cultured mammalian cells (Wu et al. 2014). In both cases, the pulsatile contractility of these actomyosin networks generates pulsatile movement of Ecadherin clusters at those junctions. Interestingly, despite their evolutionarily distant cells of origin, these pulsatile systems are distinguished by periods of similar order $(\sim 100 \mathrm{sec})$, suggesting that they might reflect some common mechanism (Baird et al. 2016). The basis of pulsatility may arise from oscillations in upstream regula- 
tors, such as RhoA signaling (Munjal et al. 2015; Mason et al. 2016), as well as properties intrinsic to the actomyosin apparatus itself (Baird et al. 2016), such as F-actin turnover (Wu et al. 2014).

In other circumstances, actomyosin may generate a tonic contractility that is reflected in tension within cell-cell junctions (FernandezGonzalez et al. 2009; Ratheesh et al. 2012; Rauskolb et al. 2014). This is seen in polarized epithelial cells, particularly those that display a zonula adherens (ZA). This is a specialized form of adherens junctions (Meng et al. 2008), where stabilized E-cadherin concentrates in a ring at the apical-lateral interface between cells (Priya et al. 2013), accompanied by actomyosin bundles that run parallel to the ZA (Smutny et al. 2010). In these cells, junctional tension is greatest at the ZA, compared with the contractile cortex of the lateral junctions below the ZA ( Wu et al. 2014). Contractility can also generate patterns of flow in the cell cortex to move molecules (Munro et al. 2004) and, indeed, basal-to-apical flow of cadherins has been observed at junctions between some epithelial cells (Kametani and Takeichi 2007). Together, these observations suggest that the organization of the actomyosin system can itself influence the patterns of force that it exerts on cadherin junctions.

Of note, the E-cadherin adhesion system itself plays an important role in the biogenesis of junctional actomyosin, as its level is decreased when E-cadherin is depleted (Smutny et al. 2010; Ratheesh et al. 2012). This impact of E-cadherin appears to reflect multiple mechanisms that are coordinated for actomyosin biogenesis, including signals that activate NMII and stimulation of cortical actin assembly (Kovacs et al. 2011; Michael et al. 2016). These mechanisms have been reviewed in detail elsewhere (Ratheesh and Yap 2012; Priya and Yap 2015). However, for the purposes of our discussion, we would highlight two points. First, cadherin-based junctions can be usefully understood as integrated systems that couple adhesion receptors with the dynamic cytoskeleton, rather than combinations of independent adhesion and cytoskeleton. Second, the junctional actomyosin cytoskeleton is dynamic even in morphologically quiescent epithelia
(Yamada et al. 2005; Smutny et al. 2010). Thus, the steady-state actomyosin that is seen at the junctional cortex is sustained by constant actin assembly and NMII activation. Such a dynamic steady-state system can then potentially be tuned in response to physiological signals, including mechanical stimuli.

Patterns of contractile force at junctions are also subject to differential spatial organizations at the supracellular level. For example, junctional tension appears to be isotropic in established epithelia that are morphogenetically stable. In contrast, morphogenetically active tissues can establish planar-polarized patterns of contractility. This is seen in the formation of the ventral furrow during gastrulation in the early Drosophila embryo, which entails the developmentally regulated induction of pulsatile medialapical actomyosin networks in the mesodermal precursors (Martin et al. 2009; Martin et al. 2010). In other circumstances, planar-polarized differences in cadherin turnover have been implicated in patterning contractile stresses (Levayer and Lecuit 2013).

An interesting example of local anisotropy in junctional stress occurs when epithelial cells undergo apoptosis. Apoptotic cells become hypercontractile when effector caspases cleave ROCK1 to generate a constitutively active form of the enzyme (Coleman et al. 2001; Sebbagh et al. 2001). When this occurs in epithelia, the cells immediately adjacent to the apoptotic cell elongate, as if being pulled by the contractile cell, and vinculin levels, an index of junctional tension (Hara et al. 2016), increase at the junctions between the apoptotic cells and their neighbors (Lubkov and Bar-Sagi 2014). This implied that contractile stresses might be transferred from apoptotic cells to their neighbors through cell-cell junctions. Indeed, E-cadherin was necessary for these morphological changes to occur (Lubkov and Bar-Sagi 2014).

\section{Other Cellular Sources of Mechanical Stress}

Contractility is not the only form of cellular force than can potentially impinge on cell-cell adhesion molecules. Osmotic forces are responsible for the growth of luminal structures, such 
A.S. Yap et al.

as the liver (Li et al. 2016) and the gut (Bagnat et al. 2007). This pressure can potentially be transmitted directly to apical cell-cell junctions and indirectly to junctions via the cortex of the apical cell surface. When epithelial cells migrate collectively, intercellular stresses also increase at the junctions that couple the moving cells together (Bazellieres et al. 2015). The magnitude of these forces and whether they elicit cellular responses remains to be determined.

Finally, it is important to note that mechanical equilibrium mandates that localized differences in force are transmitted some distances within tissues (Barry et al. 2015; Das et al. 2015). The distances over which forces propagate are entirely determined by the viscoelastic properties of the tissue and are expected to decay over a few cell diameters. However, these viscoelastic properties are substantially determined by the cytoskeleton (Howard et al. 2011). Thus, the propagation distance can be modified if cells actively react to changes in force by altering the mechanical properties of their cytoskeletons. For example, active softening of the cytoskeleton would be expected to decrease the propagation distance, whereas it would be increased if cells stiffen. Similarly, if force were to induce a contractile response in neighboring cells (Michael et al. 2016), this would add another localized source of mechanical constraint to amplify the mechanical signal and promote its large-scale propagation. This highlights the central role that cellular contractility potentially plays in the generation of, and response to, cellular forces within tissues.

\section{MEASURING FORCES AT CEL-CELL CONTACTS}

The mechanobiology of cell-cell junctions has been made possible by recent advances in tools that can measure forces in cells and whole organisms. A range of reagents and assays are now available for the investigator to use, which have been considered in detail in recent reviews (see Trier and Davidson 2011; Sugimura et al. 2016). Here, we will focus on some conceptual aspects that pertain to the forces that have to be measured and the nature of the assays themselves.

\section{A Conceptual Preamble}

The adhesion strength of adherens junctions is determined by many factors. In addition to the binding energy of trans-bonded cadherin receptors, it is increasingly clear that a large part of the adhesion strength is regulated by the properties of the underlying cytoskeleton and its coupling to cadherins (Fig. 1). A detailed analysis of how cortical dynamics and mechanics affect adhesion has still to be performed. However, we would like to highlight two important physical considerations that are often overlooked in the literature.

First, at the molecular level the mechanical force acting on a bond to disrupt it or on a protein to induce a conformational change is very often exerted at a distance across the cytoplasm or the plasma membrane. The amplitude and direction of this force will depend on whether the mechanical properties of the medium tend to localize mechanical stress or redistribute it over many molecules. For example, mechanical force can be transmitted from the cytoskeleton of one cell to the next via the transbinding of E-cadherin. This mechanical coupling occurs as long as the bond lasts. Of note, the traction force exerted by the cytoskeleton on an E-cadherin molecule can have components that are "orthogonal" to the junction, or "parallel" to the junction. The relative magnitude of these components will influence their impact on junctions, and will depend on many contextual factors, such as the geometry of the actomyosin networks and the scale of analysis. The orthogonal component can be partially borne by bending of the plasma membrane and its associated membrane skeleton. Consequently, the molecular "pulling" force that the cytoskeleton exerts on the cadherin molecular complex might be only partly felt at the trans-binding domain and only partly transmitted to the other cell. Changing mechanical properties of the junctional cortex could thus increase/alter the mechanical forces that are applied to the transbinding bond even if the strength of the underlying contractility is unchanged.

Second, when mechanical tension is measured at the cell-cell interface one should un- 


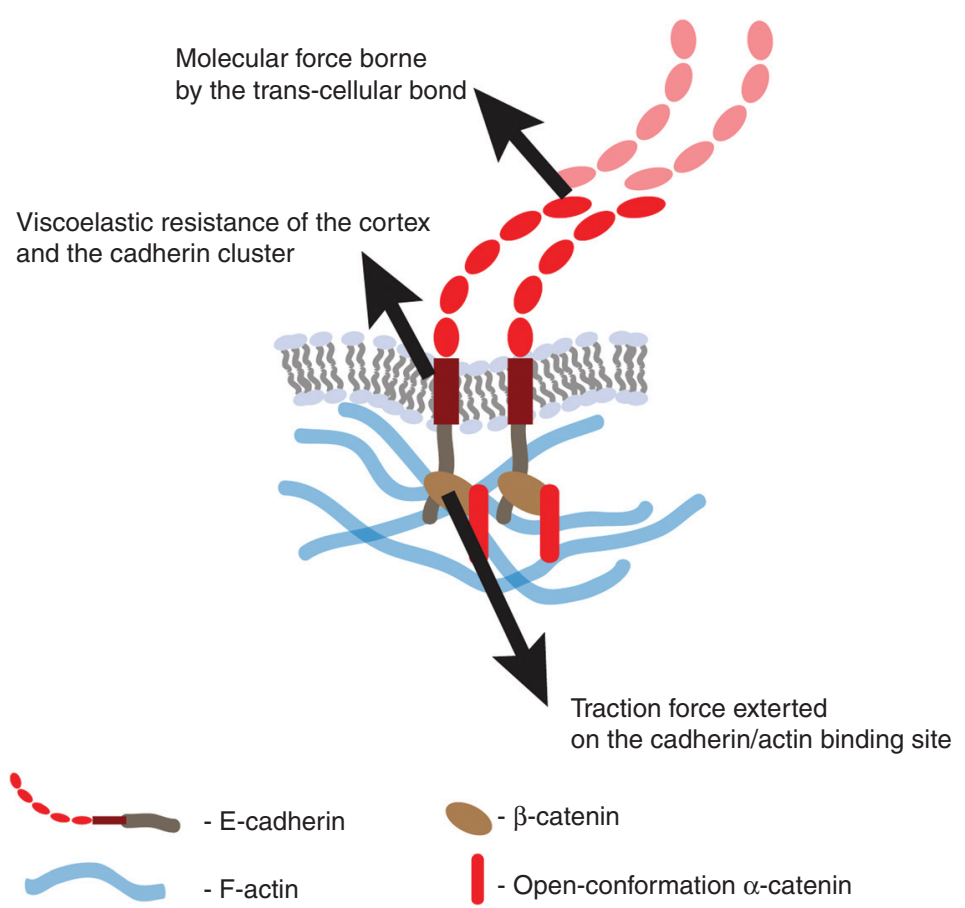

Figure 1. Molecular forces exerted on single molecular bonds. They are likely exerted on the cytoplasmic domain of E-cadherin when it is attached to F-actin networks. Molecular reporters such as Forster resonance energy transfer (FRET) sensors are expected to be sensitive to this local force. Due to its embedding in the plasma membrane and lateral clusters, the force exerted on the extracellular domain of E-cadherin and transmitted to the neighboring cells might differ significantly from the traction force applied to its intracellular domain.

derstand that a coarse grained, super-molecular view of the cell-cell contact is being adopted in the analysis. The local mechanical stress exerted on a small area of the contact also has components parallel and orthogonal to the contact surface (Fig. 2). These components approximately correspond to the average component of the molecular forces exerted at this surface. As a consequence of what we have discussed above, the stress exerted at the "contact" planes between cadherins can differ significantly from the stress at the "cortical" plane. Therefore, cadherins are unlikely to bear fully the tension of the underlying cortex. Cortical tension "along" the cellular contact should not be misinterpreted as the tension that acts "across" the contact. It also follows that the structure of the cortical Factin network that lies orthogonal or parallel to the junction has to be considered whenever possible. Therefore, when intercellular stress is experimentally reported, great care should be taken to understand exactly what quantity was measured.

\section{Techniques to Measure Mechanical Force}

With these considerations in mind, it is useful to review the range of tools that are available to measure mechanical force at cell-cell junctions, ideally within intact cells, keeping in mind the type of cellular stress that is effectively measured. These broadly fall into two categories: assays that endeavor to infer mesoscopic ( $\mu \mathrm{m}$ scale) patterns of force; and those that measure molecular level forces.

The range of mesoscopic assays for force include inference of tension from velocities of recoil after junctions are cut with lasers (Fernandez-Gonzalez et al. 2009; Ratheesh et al. 2012); assays for intercellular stresses that are inferred from measurement of traction forces (Liu et al. 2010); and inference of forces from 
A.S. Yap et al.

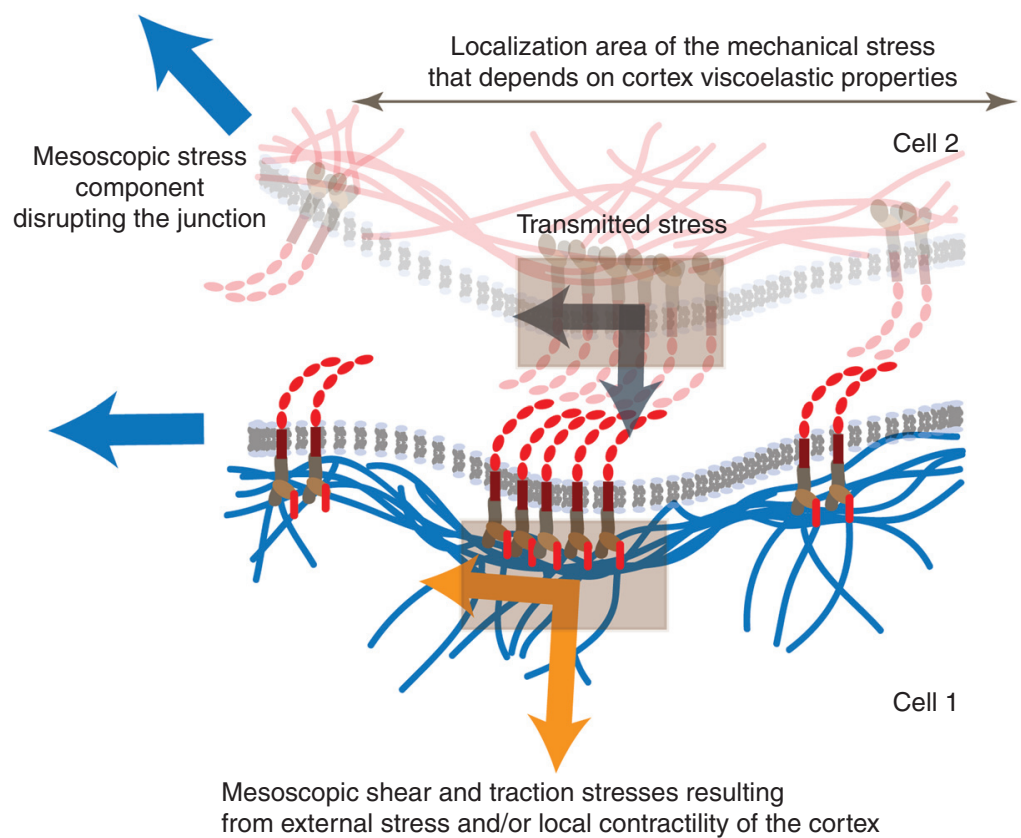

Figure 2. Molecular forces at the supramolecular scale. At a larger scale the mechanical stress generated by the contractility of the cortical actin on a junction can be decomposed into a shear stress parallel to the junction and into a tensional stress orthogonal to the junction (orange branched arrow). These two stresses are expected to have different effects on the junction behavior. The transmission of these stresses to and across the junction (dark gray branched arrow) also depends on the local geometry and rheological properties of the junction. An external disruptive stress (blue arrows), for example, is borne differently by the junction cadherins depending the angle of disruption. The viscoelastic properties of the cortex, the rate at which the stress is exerted, and the lability of the cadherin trans bonds, will also regulate the spatial extent over which the disruptive stress is supported.

changes in junctional morphology (Otani et al. 2006) or direct measurement of the elasticity of the junctional membrane using laser tweezers (Bambardekar et al. 2015). These can be complemented with other approaches that address the mechanical properties of junctions, including atomic force microscopy (Harris et al. 2014), and estimation of hydrodynamic coupling using fiduciary markers, such as beads microinjected into cells (He et al. 2014). These are powerful approaches, but it is important to emphasize that they generally carry some assumptions. For example, to infer tension from recoil measurements, it is necessary to correct for potential viscoelastic dissipation, an approach that is typically performed by using mechanical models (Fernandez-Gonzalez et al. 2009). Similarly, inference of intercellular stress from the imbalance of the stress measured on the substrate (traction forces) relies on the assumption that viscous drag caused by cell motion is negligible and that mechanical forces must therefore equilibrate. Finally, it is important to emphasize that these mesoscopic assays do not provide direct information on the molecules that are bearing force. Even when an adhesion molecule, such as green fluorescent protein (GFP)-tagged E-cadherin, is used to monitor junctional recoil after laser ablation, any changes in tension reflect changes in the membrane structure within which E-cadherin is embedded, and not necessarily changes in the molecular-level force acting on E-cadherin itself.

An important advance in efforts to monitor specific molecular level forces has come from the development of genetically encodable tension sensors that use Forster resonance energy transfer (FRET) (Grashoff et al. 2010). These 
sensors typically involve FRET fluorophore pairs that are separated by a spring-like linker peptide that stretches or unfolds when mechanical force is applied (Freikamp et al. 2016). Such tension sensors have now been introduced into cadherins (Borghi et al. 2012; Conway et al. 2013; Cai et al. 2014) and some of their cytoskeletal linker proteins, such as vinculin (Leerberg et al. 2014) and $\alpha$-catenin (Kim et al. 2015; Acharya et al. 2017). They have been used to probe forces at junctions, both in cell culture (Borghi et al. 2012; Conway et al. 2013; Leerberg et al. 2014) and in whole organisms (Cai et al. 2014). However, current sensors have their limitations. Specific molecules within adhesions may be uncoupled from the forces to which the adhesion complex as a whole is subject (Grashoff et al. 2010). The dynamic range of these sensors is limited by the spring properties of the linker peptides chosen, although recent refinements have sought to improve this (Freikamp et al. 2016). Finally, it should be noted that even though these sensors provide a level of molecular specificity that is not readily available with coarse-grained mesoscopic assays, efforts to infer the cellular level of stress from molecular level assays can be prone to error for the reasons detailed above. Therefore, it is best to use a number of complementary assays to measure forces wherever possible.

\section{MECHANISMS FOR MECHANICAL FORCE SENSING}

Understanding junctional force-sensing requires that we identify the molecular and cellular mechanisms that mediate two core processes: (1) Mechanosensitivity: mechanisms that directly sense changes in mechanical force, often through changes in molecular conformation. Although this is a precondition for force to be sensed, all proteins deform under mechanical stress. Therefore, to identify the biologically relevant events, it is also necessary to determine the mechanosensors that are responsible for: (2) Mechanotransduction: the signaling pathways that elicit cellular responses. The latter condition is important to emphasize: the demonstration that a protein is under force does not necessarily mean that its function is altered. For adherens junctions, current work identifies roles for the cadherin molecular complex and also the actomyosin cortex with which it associates. It should be noted that much current effort is devoted to identifying mechanisms that sense "increases" in force at junctions. However, "decreases" in force at junctions also occur (Conway et al. 2013), although less is known about whether these are actively sensed by cells. Other membrane components found at or near adherens junctions, such as ion channels (Martinac 2004) and caveolae (Sinha et al. 2011), can as well support mechanosensing and mechanotransduction.

\section{Force-Sensing by the Cadherin Molecular Complex}

As proximal load-bearing elements, it is parsimonious to hypothesize that cadherins and/or their associated proteins (the cadherin molecular complex) might be responsible for force sensing. Indeed, fusion proteins bearing FRETbased TS modules revealed that E-cadherin and VE-cadherin experience tension in epithelial and endothelial monolayer cultures, respectively (Borghi et al. 2012; Conway et al. 2013). This constitutive tension found in steady-state populations reflects cellular contractility, being reduced when actomyosin activity is inhibited. Furthermore, tension was detected across Drosophila epithelial (DE)-cadherin in migrating clusters of border cells, which are found in the fly egg chamber (Cai et al. 2014). This molecular level tension is likely to reflect the role of cadherin in bearing the load when adhesion mechanically couples together the actomyosin cytoskeletons of neighboring cells (Borghi et al. 2012; Conway et al. 2013) and/or the traction forces when border cells migrate on cadherin adhesions (Cai et al. 2014). However, there is no evidence, to date, that tension alters the molecular function of the cadherins themselves. Although this view may yet be altered with new information, current data suggest that cadherins principally serve to bear and transmit forces.

Instead, the search for junctional molecules whose function is altered by force has focused 
A.S. Yap et al.

on other components of the cadherin molecular complex (Fig. 3), notably $\alpha$-catenin and its interaction partner, vinculin, which both bear tension within cells, as shown using molecular tension reporters (Leerberg et al. 2014; Kim et al. 2015; Acharya et al. 2017). Importantly, recent studies have shown molecular properties of $\alpha$-catenin that are altered by mechanical force, notably its capacity to interact with other proteins. As discussed in greater detail by Mège and Ishiyama (2017), $\alpha$-catenin can directly bind F-actin as well as a host of other cytoskeletal and signaling molecules. However, although isolated $\alpha$-catenin can readily associate with F-actin (Rimm et al. 1995), this interaction could not be detected when $\alpha$-catenin was incorporated into the cadherin-catenin complex (Drees et al. 2005; Yamada et al. 2005) until force was applied to the complex (Buckley et al. 2014). Similarly, the use of magnetic tweezers showed that tension across a purified $\alpha$ catenin fragment promoted its direct binding to vinculin (Yao et al. 2014), confirming the cellular observation that vinculin recruitment to junctions is sensitive to actomyosin activity (Wen et al. 2009; le Duc et al. 2010; Yonemura et al. 2010; Leerberg et al. 2014). Therefore, at least two of the major protein-binding interactions of $\alpha$-catenin are tension-sensitive.

How force regulates these protein-protein binding events is best understood for the association of $\alpha$-catenin with vinculin. This interaction involves a central region of the $\alpha$-catenin molecule, consisting of the vinculin-binding domain (VBD) and an adjacent so-called modulatory domain. Structural studies have revealed that the $\alpha$-catenin molecule can adopt a closed, autoinhibited conformation that is predicted to prevent the VBD from interacting with vinculin (Choi et al. 2012; Rangarajan and Izard 2012; Ishiyama et al. 2013). The first clue that this conformation might be regulated by force came from the observation that a cryptic epitope located in this autoinhibited region of

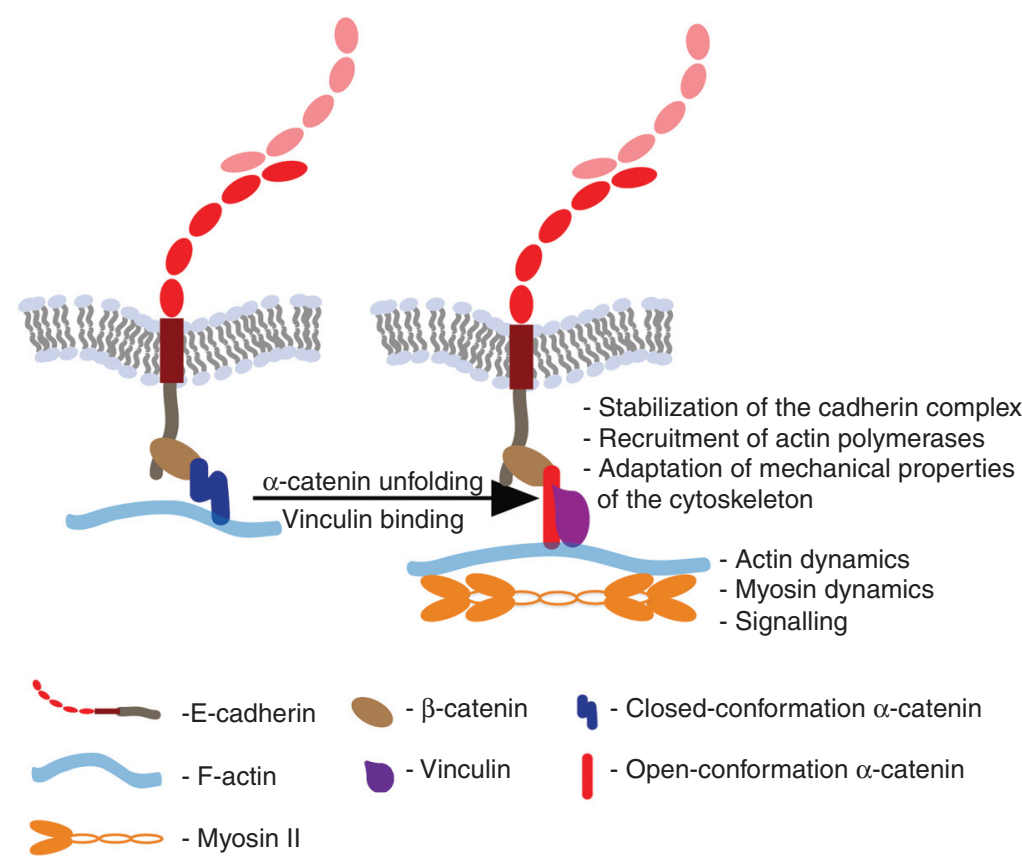

Figure 3. A model for mechanotransduction through the cadherin-catenin complex. Tensional forces are exerted on cadherins and their associated proteins when adhesion couples the contractile cortices of neighboring cells together. Tension causes $\alpha$-catenin to unfold promoting association with vinculin. The formation of this force-sensitive complex has multiple sequelae, mediated by changes in cytoskeletal dynamics and cell signaling. 
$\alpha$-catenin required cellular contractility to be detected by immunocytochemistry (Yonemura et al. 2010). This suggested that contractile force might favor an open state of the $\alpha$-catenin molecule. Recently, Yao et al. (2014) used magnetic tweezers to show that the purified central domain unfurls on the application of physiological level forces $(\sim 5 \mathrm{pN})$, comparable to what can be generated by a single myosin motor (Finer et al. 1994). How force promotes binding of F-actin to $\alpha$-catenin that is incorporated into a cadherin-catenin complex is not understood in such molecular detail. Kinetic modelling was consistent with a two-state catch-bond model in which force promoted strong binding interaction between the cadherin-catenin complex and F-actin (Buckley et al. 2014).

Its capacity to promote protein - protein interactions provides one paradigm for how force can alter the function of the cadherin molecular complex. This may ramify in several reported ways (Fig. 3). First, force-regulated proteinprotein interactions may influence the stability of the cadherin molecular complex itself. Thus, the force-induced binding of vinculin tended to inhibit refolding of the $\alpha$-catenin central fragment (Yao et al. 2014), thereby stabilizing its "open" conformation. This could potentiate its binding to vinculin and perhaps other associated proteins. Vinculin can also adopt an autoinhibited conformation (Johnson and Craig 1995; Bakolitsa et al. 2004), but its binding affinity for $\alpha$-catenin (Yao et al. 2014) is greater than that of the intramolecular interaction that is responsible for its autoinhibited conformation (Bakolitsa et al. 2004). Binding $\alpha$-catenin might then also "activate" vinculin to allow it to associate with its interaction partners (Chen et al. 2006).

Therefore, force-dependent stabilization of protein-protein interactions within the cadherin molecular complex might not only stabilize the complex, but also promote downstream functional consequences. One example is actin assembly at junctions, which is sensitive to contractility, being decreased when myosin was inhibited and increased when contractility was stimulated (Leerberg et al. 2014). One mechanism in this case entailed Enabled/vasodilator- stimulated phosphoprotein (Ena/VASP) proteins, which can bind directly to vinculin and were corecruited to junctions with vinculin in response to contractility. Ena/VASP proteins are barbed-end actin polymerases that promote elongation of actin filaments after they have been nucleated (Hansen and Mullins 2010). By this mechanism, mechanotransduction through $\alpha$-catenin can modulate both cortical actin filament dynamics as well as the physical association of cadherin complexes with F-actin (Buckley et al. 2014).

On application of force, vinculin at cadherin adhesions also undergoes phosphorylation at tyrosine 822 , which was necessary for the stable incorporation of vinculin into the cadherin molecular complex, junction formation, and subsequent cortical stiffening (Bays et al. 2014). Phosphorylation was mediated by the Abelson tyrosine kinase, which is found at cell-cell junctions (Tamada et al. 2012), and whose activity was enhanced substantially when force was applied to E-cadherin (Bays et al. 2014) by a pathway that entailed the stress-induced activation of AMP kinase (Bays et al. 2017). Therefore, force-induced changes in cell signaling may provide an additional paradigm for mechanotransduction to occur. The activation of signaling pathways also provides the capacity for mechanotransduction at cell-cell adhesions to propagate more extensively within cells. For example, mechanical stimulation of E-cadherin led to an increase in the traction forces that cells exerted on extracellular matrix (Muhamed et al. 2016). This cross-talk entailed the activation of integrin adhesion receptors and appeared to be mediated by phosphoinositide-3-kinase, which was activated when force was applied to cadherin adhesions.

Force-Sensing by the Junctional Cytoskeleton

The cell cytoskeleton itself contains many mechano-sensitive elements, ranging from regulators of actin dynamics to myosin motors. Modulation of the actin cytoskeleton by application of force on cadherin-based adhesions was first shown for E-cadherin (le Duc et al. 2010) and subsequently confirmed for VE-cadherin (Barry et al. 2015). These experiments 
A.S. Yap et al.

used adhesions made when magnetic beads coated with functional cadherin ligands bound to the dorsal surfaces of cells. Force was applied by twisting the beads in an oscillatory magnetic field. This caused the cells to stiffen, a process that required a functional cadherin ligand and intact actomyosin (le Duc et al. 2010). Of note, stiffening was evident within $10 \mathrm{sec}$ (le Duc et al. 2010), a rapidity that might reflect a direct cytoskeletal response when force was applied to the cadherin.

Such an early-immediate response could occur if force were to modulate the binding affinities or turnover of the cytoskeletal elements themselves. Indeed, recent work has identified a number of ways in which this might occur. Actin filaments themselves have been proposed to deform under mechanical stimulation, with the effect of altering the binding and/or unbinding rates of actin-binding proteins (Galkin et al. 2012; Morimatsu et al. 2012; Galkin et al. 2015). Tension may also promote formin-dependent actin filament assembly (Kozlov and Bershadsky 2004) and antagonize binding of the actin-disassembly protein, cofilin (Hayakawa et al. 2011). This could mediate early-immediate changes in F-actin dynamics when the cadherin-bound actin cytoskeleton is subjected to mechanical force, which might complement slower-acting mechanisms, such as the force-activated recruitment of vinculin and Ena/VASP proteins (Leerberg et al. 2014), discussed earlier.

Myosins are another broad class of actinbinding proteins whose kinetics of association with actin filaments are sensitive to mechanical force (De la Cruz and Ostap 2004). Furthermore, it is increasingly apparent that, in addition to its role in contractility, myosin II can also serve as a scaffold for other cytoskeletal and signaling proteins (Ma et al. 2007; Kuo et al. 2011; Billington et al. 2015). These include ROCK1, which stabilizes RhoA GTPase signaling at the epithelial ZA when it is anchored by myosin IIA (Priya et al. 2015). Such forms of mechanochemical feedback, which are elicited when the binding of myosin II to junctional actin filaments is stabilized, then provide another paradigm for cells to sense forces applied to cadherins.

\section{FUNCTIONAL CONSEQUENCES OF CADHERIN-BASED MECHANOTRANSDUCTION}

We are now beginning to understand the functional consequences of force-sensing and mechanotransduction at cadherin cell-cell junctions. Classical cadherins affect many aspects of cell and tissue behavior, encompassing cell-cell cohesion and morphogenesis, control of cell migration, cell differentiation, and proliferation. To what extent these reflect the impact of mechanosensing at junctions remains to be determined. Here, we focus on two emerging areas: regulation of the cytoskeleton and cellular mechanics; and control of population number in tissues. These emphasize that, by utilizing distinct signaling pathways, junctional mechanotransduction can elicit biological effects that operate on substantially different time scales.

\section{Early Proximal Consequences: Adaptation of Cortical Mechanics}

As discussed earlier, many of the early-immediate responses to junctional stress involve the cytoskeleton itself. These responses could constitute a mode of mechanical feedback that would adapt the mechanical properties of the cytoskeleton (viscosity, elasticity) to the application of force. This can, in turn, influence adhesion. For example, increasing myosin II-based contractility in isolated cell doublets stabilized cortical Factin turnover and immobilized E-cadherin in clusters at the junction (Engl et al. 2014). Similarly, myosin II stabilizes E-cadherin at the ZA in epithelial monolayers (Ratheesh et al. 2012). These coordinate changes in cytoskeleton and adhesion may influence the viscous dissipation of mechanical stress, and ultimately influence whether junctional integrity is preserved in the face of such stress.

To understand how regulated cortical mechanics can influence junctional integrity, it is useful to consider cell-cell adhesion strength in terms of the energy that is required to separate two objects. Adhesion energy is not an intrinsic property of the adherent elements alone, but depends on how the disrupting stress is applied and 
the rate at which the disruption occurs. Therefore, adhesion energy is critically influenced by the rheological properties of the adhesive interface and the mechanical properties of the material (deformability, elasticity, viscosity) that dictate the spatial distribution of the local stress that is applied to the chemical bonds that eventually have to bear the force. A classic example is a double-sided tape that can hold heavy weight, yet is still easily detachable when peeled from its edges. In this light, stress-induced reinforcement of the junctional cytoskeleton might increase its rigidity and decrease how readily cell-cell contacts are peeled away from one another.

The localization of stress further depends on the local dissipation of the mechanical stimulation (e.g., contractility) as well as on the binding/unbinding dynamics of linkers (Mayumi et al. 2016). When bonds are labile, their ability to reform also plays an important role in establishing the amount of mechanical force needed to disrupt a junction. For junctions, these linkers include the adhesive cadherin bonds themselves: enhanced cadherin clustering in response to contractility can increase cell adhesion (Smutny et al. 2010), thereby reinforcing resistance to detachment. Therefore, the response of junctions to stress is not simply the outcome of applying disruptive forces to passive resistive bonds, but is further conditioned by how mechanical feedback affects the rheology of the adhesive interface.

Adaptation of cortical mechanics may contribute to a number of morphogenetic circumstances in which junctional integrity must be preserved despite large mechanical loads associated with tissue deformation. These include collective cell migration of epithelia, which is associated with increased intercellular stresses (Bazellieres et al. 2015), and cortical flows of cadherin and actin in astrocytes (Peglion et al. 2014). Another interesting example of cortical adaptation is seen in epithelial apoptosis. As noted above, apoptotic cells become hypercontractile and, when this occurs in epithelia that are coupled together by E-cadherin, forces from the apoptotic cells can be transferred to their neighbors (Lubkov and Bar-Sagi 2014). Characteristically, those neighbor cells respond by assembling a contractile ring at their junction with the apoptotic cell (Rosenblatt et al. 2001; Slattum et al. 2009; Michael et al. 2016). Ultimately, this leads to the extrusion of the apoptotic cell, a homeostatic process that allows injured cells to be eliminated from an epithelium without disturbing the integrity of its barrier (Rosenblatt et al. 2001). The formation of the contractile ring within the neighboring cells reflects the action of several processes, including intercellular signaling by sphingosine-1-phosphate (Gu et al. 2011) and dynamic microtubules that regulate RhoA signaling in the neighbor cells (Slattum et al. 2009). The neighbor cell contractile ring can also be induced to form when contractility is enhanced without cell injury by expression of a constitutively active ROCK mutant (Lubkov and Bar-Sagi 2014; Michael et al. 2016). Therefore, junctional mechanotransduction may also contribute to stimulating formation of the contractile ring.

\section{Lasting Consequences: Control of Cell Proliferation by Transcription}

Cell-cell adhesion has been implicated in the control of cell proliferation through a variety of potential pathways (reviewed in greater detail in Chiasson-MacKenzie and McClatchey 2017). One of these is Hippo signaling, which can integrate diverse signals to control cell proliferation and organ size (Tapon and Harvey 2012). As discussed by Karaman and Halder (2017), the Hippo pathway serves to inhibit cell proliferation by blocking the nuclear translocation of the transcriptional regulator, Yorkie (in Drosophila in which the pathway was first discovered, Yap1 in mammals). Key to this pathway is the serine/threonine protein kinase Warts (Wts in Drosophila, LATS in mammals), which phosphorylates Yorkie/Yap1 to prevent it from signaling. A number of components of the Hippo pathway have been found at cell-cell junctions, including Yap1 itself (Kim et al. 2011). Cellular contractility was also found to influence signaling by Yorkie, Yap1, and its paralog, Taz (Dupont et al. 2011).

Recent studies in the Drosophila wing disc have revealed that junctional tension decreases 
A.S. Yap et al.

when cell proliferation slows (Rauskolb et al. 2014). Conversely, increasing cytoskeletal contractility by driving Rho kinase signaling or myosin II promoted cell proliferation. This mechanical regulation of cell proliferation was attributable to Yorkie signaling. Similarly, mechanical stretch of mammalian epithelial (MDCK) monolayers activated Yap1 to allow cell-cycle entry for cell proliferation (BenhamPyle et al. 2015). A key to the underlying mechanism in the fly was the observation that Wts became recruited to adherens junctions when cellular contractility was stimulated. Presumably, this segregated it from its target, allowing Yorkie to become disinhibited. Junctional recruitment of Wts was mediated by the scaffolding protein, Ajuba, a negative regulator of $\mathrm{Wts}$ that binds directly to it. Ajuba, in turn, was recruited to junctions by $\alpha$-catenin (Marie et al. 2003; Rauskolb et al. 2014). Overall, this suggested a mechanism in which cytoskeletal tension acting on junctions promotes the association of Ajuba with $\alpha$-catenin, leading to the recruitment of $\mathrm{Wts}$.

This mechano-sensitive pathway may explain an interesting paradox in epithelial homeostasis. Often, growing tissues, such as the Drosophila wing disc, display homogeneous patterns of cell proliferation, despite an inhomogeneous expression of growth factors (Pan et al. 2016). This raises the question of why regions that are located close to the site of growth factor secretion and thus experience higher concentrations of growth factors, do not proliferate more rapidly than areas located further away. However, the decrease in junctional and tissue tension that has been observed to accompany overproliferation would be predicted to trigger negative feedback by releasing Wts from adherens junctions (Rauskolb et al. 2014). Indeed, junctional levels of both Ajuba and Wts were reduced in regions that were relatively hyperproliferative, coinciding with reduced Yorkie signaling (Pan et al. 2016). Conversely, manipulations that interdicted the mechano-sensitive regulation of Ajuba/Wts and allowed Yorkie signaling to persist caused local tissue overproliferation and distorted tissue architecture (Pan et al. 2016). Therefore, regulation of Ajuba/Wts signaling in response to junctional mechanotransduction may balance local proliferation rates to maintain tissue organization.

There are likely to be many other ways in which junctional mechanotransduction can influence cell proliferation. Thus, in mammalian skin $\alpha$-catenin was observed to function as a negative regulator of Yap1 (Schlegelmilch et al. 2011; Silvis et al. 2011), suggesting that species and/or tissue context may critically influence the pathway that dominates; and stretching MDCK monolayers could also induce nuclear translocation of $\beta$-catenin to promote cell cycle progression (Benham-Pyle et al. 2015). Nonetheless, as the effective product of these pathways is transcriptional regulation, they provide a model for how changes in junctional forces can be translated into the longer time scales of proliferative regulation in tissues. An interesting question is whether these mechanotransduction pathways may also collaborate with the regulation of mitogenic signals to influence contact inhibition of cell proliferation.

\section{CONCLUDING REMARKS}

It seems evident that we have only just begun to appreciate the extent to which mechanical signals can mediate cell-cell communication when adhesion couples cells together into tissues. As noted above, changes in physical force have the capacity to propagate between cells, with potential directionality and length scales that are determined by the mechanical properties of those tissues. In addition, changes in force can propagate very rapidly (Howard et al. 2011), providing the opportunity to complement slower-acting chemical signals such as diffusible mediators. Progress in this area will continue to be driven by technical innovations, such as the development of new tools to measure forces, combined with multidisciplinary collaborations that bring biologists together with physicists, engineers, and theoreticians. An interesting question will be to define how much the active tension that is generated at cell-cell junctions is used for cell-cell communication. Here, we can draw an historical analogy from the study of mechanotransduction at 
focal adhesions, where contractile forces are generated on integrin-based adhesions (Geiger et al. 2009). Initially, the mechano-sensitive response of integrins was thought to principally serve to reinforce adhesion. Later, it became clear that active contractility provided a force that allowed integrin mechanotransduction to be used by cells to sense the mechanical properties of their extracellular matrix (Geiger et al. 2009). This had profound consequences for regulating cell proliferation and differentiation in health and disease (Yu et al. 2011). Whether a similar paradigm pertains for cell-cell adhesions will be an exciting question for the future.

\section{ACKNOWLEDGMENTS}

A.S.Y. was supported by the National Health and Medical Research Council of Australia (1044041); K.D. is the recipient of an International Postgraduate Research Scholarship and a UQ Centennial Scholarship; and V.V. acknowledges support from the Mechanobiology Institute of Singapore.

\section{REFERENCES}

* Reference is also in this subject collection.

Acharya BR, Wu SK, Lieu ZZ, Parton RG, Grill SW, Bershadsky AD, Gomez GA, Yap AS. 2017. Mammalian Diaphanous 1 mediates a pathway for E-cadherin to stabilize epithelial barriers through junctional contractility. Cell Rep 18: 2854-2867.

Bagnat M, Cheung ID, Mostov KE, Stainier DY. 2007. Genetic control of single lumen formation in the zebrafish gut. Nat Cell Biol 9: 954-960.

Baird MA, Billington N, Wang A, Adelstein RS, Sellers JR, Fischer RS, Waterman CM. 2016. Local pulsatile contractions are an intrinsic property of the myosin $2 \mathrm{~A}$ motor in the cortical cytoskeleton of adherent cells. Mol Biol Cell 28: $240-251$.

Bakolitsa C, Cohen DM, Bankston LA, Bobkov AA, Cadwell GW, Jennings L, Critchley DR, Craig SW, Liddington RC. 2004. Structural basis for vinculin activation at sites of cell adhesion. Nature 430: 583-586.

Bambardekar K, Clement R, Blanc O, Chardes C, Lenne PF. 2015. Direct laser manipulation reveals the mechanics of cell contacts in vivo. Proc Natl Acad Sci 112: 1416-1421.

Barry AK, Wang N, Leckband DE. 2015. Local VE-cadherin mechanotransduction triggers long-ranged remodeling of endothelial monolayers. J Cell Sci 128: 1341-1351.

Bays JL, Peng X, Tolbert CE, Guilluy C, Angell AE, Pan Y, Superfine R, Burridge K, Demali KA. 2014. Vinculin phosphorylation differentially regulates mechanotransduction at cell-cell and cell-matrix adhesions. J Cell Biol 205: 251-263.

Bays JL, Campbell HK, Heidema C, Sebbagh M, DeMali KA. 2017. Linking E-cadherin mechanotransduction to cell metabolism through force-mediated activation of AMPK. Nat Cell Biol 19: 724-731.

Bazellieres E, Conte V, Elosegui-Artola A, Serra-Picamal X, Bintanel-Morcillo M, Roca-Cusachs P, Munoz JJ, SalesPardo M, Guimera R, Trepat X. 2015. Control of cell-cell forces and collective cell dynamics by the intercellular adhesome. Nat Cell Biol 17: 409-420.

Benham-Pyle BW, Pruitt BL, Nelson WJ. 2015. Cell adhesion. Mechanical strain induces E-cadherin-dependent Yap1 and $\beta$-catenin activation to drive cell cycle entry. Science 348: 1024-1027.

Billington N, Beach JR, Heissler SM, Remmert K, GuzikLendrum S, Nagy A, Takagi Y, Shao L, Li D, Yang Y, et al. 2015. Myosin 18A coassembles with nonmuscle myosin 2 to form mixed bipolar filaments. Curr Biol 25: 942-948.

Borghi N, Sorokina M, Shcherbakova OG, Weis WI, Pruitt BL, Nelson WJ, Dunn AR. 2012. E-cadherin is under constitutive actomyosin-generated tension that is increased at cell-cell contacts upon externally applied stretch. Proc Natl Acad Sci 109: 12568-12573.

Buckley CD, Tan JL, Anderson KL, Hanein D, Volkmann N, Weis WI, Nelson WJ, Dunn AR. 2014. The minimal cadherin-catenin complex binds to actin filaments under force. Science 346: 1254211-1254218.

Cai D, Chen SC, Prasad M, He L, Wang X, Choesmel-Cadamuro V, Sawyer JK, Danuser G, Montell DJ. 2014. Mechanical feedback through E-cadherin promotes direction sensing during collective cell migration. Cell 157: 1146-1159.

Campinho P, Behrndt M, Ranft J, Risler T, Minc N, Heisenberg CP. 2013. Tension-oriented cell divisions limit anisotropic tissue tension in epithelial spreading during zebrafish epiboly. Nat Cell Biol 15: 1405-1414.

Chen H, Choudhury DM, Craig SW. 2006. Coincidence of actin filaments and talin is required to activate vinculin. $J$ Biol Chem 281: 40389-40398.

* Chiasson-MacKenzie C, McClatchey AI. 2017. Cell-cell contact and receptor tyrosine kinase signaling. Cold Spring Harb Perspect Biol doi: 10.1101/cshperspect.a029215.

Choi HJ, Pokutta S, Cadwell GW, Bobkov AA, Bankston LA, Liddington RC, Weis WI. 2012. $\alpha$ E-catenin is an autoinhibited molecule that coactivates vinculin. Proc Natl Acad Sci 109: 8576-8581.

Clark K, Langeslag M, Figdor CG, van Leeuwen FN. 2007. Myosin II and mechanotransduction: A balancing act. Trends Cell Biol 17: 178-186.

Coleman ML, Sahai EA, Yeo M, Bosch M, Dewar A, Olson MF. 2001. Membrane blebbing during apoptosis results from caspase-mediated activation of ROCK I. Nat Cell Biol 3: 339-345.

Conway DE, Breckenridge MT, Hinde E, Gratton E, Chen CS, Schwartz MA. 2013. Fluid shear stress on endothelial cells modulates mechanical tension across VE-cadherin and PECAM-1. Curr Biol 23: 1024-1030. 
A.S. Yap et al.

Das T, Safferling K, Rausch S, Grabe N, Boehm H, Spatz JP 2015. A molecular mechanotransduction pathway regulates collective migration of epithelial cells. Nat Cell Biol 17: 276-287.

De la Cruz EM, Ostap EM. 2004. Relating biochemistry and function in the myosin superfamily. Curr Op Cell Biol 16: 61-67.

Drees F, Pokutta S, Yamada S, Nelson WJ, Weis WI. 2005. $\alpha$ Catenin is a molecular switch that binds E-cadherin- $\beta$ catenin and regulates actin-filament assembly. Cell $\mathbf{1 2 3}$ 903-915.

Dupont S, Morsut L, Aragona M, Enzo E, Giulitti S, Cordenonsi M, Zanconato F, Le Digabel J, Forcato M, Bicciato S, et al. 2011. Role of YAP/TAZ in mechanotransduction. Nature 474: 179-183.

Ebrahim S, Fujita T, Millis BA, Kozin E, Ma X, Kawamoto S Baird MA, Davidson M, Yonemura S, Hisa Y, et al. 2013. NMII forms a contractile transcellular sarcomeric network to regulate apical cell junctions and tissue geometry. Curr Biol 23: 731-736.

Engl W, Arasi B, Yap LL, Thiery JP, Viasnoff V. 2014. Actin dynamics modulate mechanosensitive immobilization of E-cadherin at adherens junctions. Nat Cell Biol 16: 587594.

Fernandez-Gonzalez R, Simoes Sde M, Roper JC, Eaton S, Zallen JA. 2009. Myosin II dynamics are regulated by tension in intercalating cells. Dev Cell 17: 736-743.

Finer JT, Simmons RM, Spudich JA. 1994. Single myosin molecule mechanics: Piconewton forces and nanometre steps. Nature 368: 113-119.

Freikamp A, Cost AL, Grashoff C. 2016. The Piconewton force awakens: Quantifying mechanics in cells. Trends Cell Biol 26: 838-847.

Galkin VE, Orlova A, Egelman EH. 2012. Actin filaments as tension sensors. Curr Biol 22: R96-R101.

Galkin VE, Orlova A, Vos MR, Schroder GF, Egelman EH. 2015. Near-atomic resolution for one state of F-actin. Structure 23: 173-182.

Geiger B, Spatz JP, Bershadsky AD. 2009. Environmental sensing through focal adhesions. Nat Rev Mol Cell Biol 10: $21-33$.

Grashoff C, Hoffman BD, Brenner MD, Zhou R, Parsons M, Yang MT, McLean MA, Sligar SG, Chen CS, Ha T, et al. 2010. Measuring mechanical tension across vinculin reveals regulation of focal adhesion dynamics. Nature $\mathbf{4 6 6}$ : 263-266.

Gu Y, Forostyan T, Sabbadini R, Rosenblatt J. 2011. Epithelial cell extrusion requires the sphingosine-1-phosphate receptor 2 pathway. J Cell Biol 193: 667-676.

Hansen SD, Mullins RD. 2010. VASP is a processive actin polymerase that requires monomeric actin for barbed end association. J Cell Biol 191: 571-584.

Hara Y, Shagirov M, Toyama Y. 2016. Cell boundary elongation by non-autonomous contractility in cell oscillation. Curr Biol 26: 2388-2396.

Harris AR, Daeden A, Charras GT. 2014. Formation of adherens junctions leads to the emergence of a tissue-level tension in epithelial monolayers. J Cell Sci 127: 25072517.
Hayakawa K, Tatsumi H, Sokabe M. 2011. Actin filaments function as a tension sensor by tension-dependent binding of cofilin to the filament. J Cell Biol 195: 721-727.

He B, Doubrovinski K, Polyakov O, Wieschaus E. 2014. Apical constriction drives tissue-scale hydrodynamic flow to mediate cell elongation. Nature 508: 392-396.

Heisenberg CP, Bellaiche Y. 2013. Forces in tissue morphogenesis and patterning. Cell 153: 948-962.

Heissler SM, Manstein DJ. 2013. Nonmuscle myosin-2: Mix and match. Cell Mol Life Sci 70: 1-21.

Howard J, Grill SW, Bois JS. 2011. Turing's next steps: The mechanochemical basis of morphogenesis. Nat Rev Mol Cell Biol 12: 400-406.

Ishiyama N, Tanaka N, Abe K, Yang YJ, Abbas YM, Umitsu M, Nagar B, Bueler SA, Rubinstein JL, Takeichi M, et al. 2013. An autoinhibited structure of $\alpha$-catenin and its implications for vinculin recruitment to adherens junctions. J Biol Chem 288: 15913-15925.

Johnson RP, Craig SW. 1995. F-actin binding site masked by the intramolecular association of vinculin head and tail domains. Nature 373: 261-264.

Kametani Y, Takeichi M. 2007. Basal-to-apical cadherin flow at cell junctions. Nat Cell Biol 9: 92-98.

Kannan N, Tang VW. 2015. Synaptopodin couples epithelial contractility to $\alpha$-actinin-4-dependent junction maturation. J Cell Biol 211: 407-434.

* Karaman R, Halder G. 2017. Cell junctions in Hippo signaling. Cold Spring Harb Perspect Biol doi: 10.1101/cshperspect.a028753.

Kim NG, Koh E, Chen X, Gumbiner BM. 2011. E-cadherin mediates contact inhibition of proliferation through Hippo signaling-pathway components. Proc Natl Acad Sci 108: 11930-11935.

Kim TJ, Zheng S, Sun J, Muhamed I, Wu J, Lei L, Kong X, Leckband DE, Wang Y. 2015. Dynamic visualization of $\alpha$ catenin reveals rapid, reversible conformation switching between tension states. Curr Biol 25: 218-224.

Kovacs EM, Verma S, Ali RG, Ratheesh A, Hamilton NA, Akhmanova A, Yap AS. 2011. N-WASP regulates the epithelial junctional actin cytoskeleton through a non-canonical post-nucleation pathway. Nat Cell Biol 13: $934-$ 943.

Kozlov MM, Bershadsky AD. 2004. Processive capping by formin suggests a force-driven mechanism of actin polymerization. J Cell Biol 167: 1011-1017.

Kuo JC, Han X, Hsiao CT, Yates JR III, Waterman CM. 2011. Analysis of the myosin-II-responsive focal adhesion proteome reveals a role for $\beta$-Pix in negative regulation of focal adhesion maturation. Nat Cell Biol 13: 383-393.

le Duc Q, Shi Q, Blonk I, Sonnenberg A, Wang N, Leckband D, de Rooij J. 2010. Vinculin potentiates E-cadherin mechanosensing and is recruited to actin-anchored sites within adherens junctions in a myosin II-dependent manner. J Cell Biol 189: 1107-1115.

Leckband DE, de Rooij J. 2014. Cadherin adhesion and mechanotransduction. Annu Rev Cell Dev Biol 30: 291315.

Lecuit T, Yap AS. 2015. E-cadherin junctions as active mechanical integrators in tissue dynamics. Nat Cell Biol 17: 533-539. 
Lecuit T, Lenne PF, Munro E. 2011. Force generation, transmission, and integration during cell and tissue morphogenesis. Annu Rev Cell Dev Biol 27: 157-184.

Leerberg JM, Gomez GA, Verma S, Moussa EJ, Wu SK, Priya R, Hoffman BD, Grashoff C, Schwartz MA, Yap AS. 2014 Tension-sensitive actin assembly supports contractility at the epithelial zonula adherens. Curr Biol 24: 1689-1699.

Levayer R, Lecuit T. 2013. Oscillation and polarity of Ecadherin asymmetries control actomyosin flow patterns during morphogenesis. Dev Cell 26: 162-175.

Li Q, Zhang Y, Pluchon P, Robens J, Herr K, Mercade M, Thiery JP, Yu H, Viasnoff V. 2016. Extracellular matrix scaffolding guides lumen elongation by inducing anisotropic intercellular mechanical tension. Nat Cell Biol 18: 311-318.

Liu Z, Tan JL, Cohen DM, Yang MT, Sniadecki NJ, Ruiz SA, Nelson CM, Chen CS. 2010. Mechanical tugging force regulates the size of cell-cell junctions. Proc Natl Acad Sci 107: 9944-9949.

Lubkov V, Bar-Sagi D. 2014. E-cadherin-mediated cell coupling is required for apoptotic cell extrusion. Curr Biol 24: 868-874.

Ma X, Bao J, Adelstein RS. 2007. Loss of cell adhesion causes hydrocephalus in nonmuscel myosin II-B-ablated and mutated mice. Mol Biol Cell 18: 2305-2312.

Marie H, Pratt SJ, Betson M, Epple H, Kittler JT, Meek L, Moss SJ, Troyanovsky S, Attwell D, Longmore GD, et al. 2003. The LIM protein Ajuba is recruited to cadherindependent cell junctions through an association with $\alpha$ catenin. J Biol Chem 278: 1220-1228.

Martin AC, Goldstein B. 2014. Apical constriction: Themes and variations on a cellular mechanism driving morphogenesis. Development 141: 1987-1998.

Martin AC, Kaschube M, Wieschaus EF. 2009. Pulsed contractions of an actin-myosin network drive apical constriction. Nature 457: 495-499.

Martin AC, Gelbart M, Fernandez-Gonzalez R, Kaschube M, Wieschaus E. 2010. Integration of contractile forces during tissue invagination. J Cell Biol 188: 735-749.

Martinac B. 2004. Mechanosensitive ion channels: Molecules of mechanotransduction. JCell Sci 117: 2449-2460.

Mason FM, Xie S, Vasquez CG, Tworoger M, Martin AC. 2016. RhoA GTPase inhibition organizes contraction during epithelial morphogenesis. J Cell Biol 214: $603-$ 617.

Mayumi K, Guo J, Narita T, Hui CY, Creton C. 2016. Fracture of dual crosslink gels with permanent and transient crosslinks. Extreme Mechanics Lett 6: 52-59.

* Mège RM, Ishiyama N. 2017. Integration of cadherin adhesion and cytoskeleton at adherens junctions. Cold Spring Harb Perspect Biol doi: 10.1101/cshperspect.a028738.

Meng W, Mushika Y, Ichii T, Takeichi M. 2008. Anchorage of microtubule minus ends to adherens junctions regulates epithelial cell-cell contacts. Cell 135: 948-959.

Michael M, Meiring JC, Acharya BR, Matthews DR, Verma S, Han SP, Hill MM, Parton RG, Gomez GA, Yap AS. 2016. Coronin 1B reorganizes the architecture of F-actin networks for contractility at steady-state and apoptotic adherens junctions. Dev Cell 37: 58-71.

Morimatsu M, Togashi Y, Nishikawa S, Sugawa M, Iwane AH, Yanagida T. 2012. Spontaneous structural changes in actin regulate G-F transformation. PLoS ONE 7: e45864.

Muhamed I, Wu J, Sehgal P, Kong X, Tajik A, Wang N, Leckband DE. 2016. E-cadherin-mediated force transduction signals regulate global cell mechanics. J Cell Sci 129: $1843-1854$.

Munjal A, Philippe JM, Munro E, Lecuit T. 2015. A selforganized biomechanical network drives shape changes during tissue morphogenesis. Nature 524: 351-355.

Munro E, Nance J, Priess JR. 2004. Cortical flows powered by asymmetrical contraction transport PAR proteins to establish and maintain anterior-posterior polarity in the early C. elegans embryo. Dev Cell 7: 413-424.

Orr AW, Helmke BP, Blackman BR, Schwartz MA. 2006. Mechanisms of mechanotransduction. Dev Cell 10: 1120.

Otani T, Ichii T, Aono S, Takeichi M. 2006. Cdc42 GEF Tuba regulates the junctional configuration of simple epithelial cells. J Cell Biol 175: 135-146.

Pan Y, Heemskerk I, Ibar C, Shraiman BI, Irvine KD. 2016. Differential growth triggers mechanical feedback that elevates Hippo signaling. Proc Natl Acad Sci 113: E6974E6983.

Peglion F, Llense F, Etienne-Manneville S. 2014. Adherens junction treadmilling during collective migration. Nat Cell Biol 16: 639-651.

Priya R, Yap AS. 2015. Active tension: The role of cadherin adhesion and signaling in generating junctional contractility. Curr Top Dev Biol 112: 65-102.

Priya R, Yap AS, Gomez GA. 2013. E-cadherin supports steady-state Rho signaling at the epithelial zonula adherens. Differentiation 86: 133-140.

Priya R, Gomez GA, Budnar S, Verma S, Cox HL, Hamilton NA, Yap AS. 2015. Feedback regulation through myosin II confers robustness on RhoA signalling at E-cadherin junctions. Nat Cell Biol 17: 1282-1293.

Rangarajan ES, Izard T. 2012. $\alpha$-Catenin unfurls upon binding to vinculin. J Biol Chem 25: 18492-18499.

Ratheesh A, Yap AS. 2012. A bigger picture: Classical cadherins and the dynamic actin cytoskeleton. Nat Rev Mol Cell Biol 13: 673-679.

Ratheesh A, Gomez GA, Priya R, Verma S, Kovacs EM, Jiang K, Brown NH, Akhmanova A, Stehbens SJ, Yap AS. 2012. Centralspindlin and $\alpha$-catenin regulate Rho signalling at the epithelial zonula adherens. Nat Cell Biol 14: 818-828.

Rauskolb C, Sun S, Sun G, Pan Y, Irvine KD. 2014. Cytoskeletal tension inhibits Hippo signaling through an Ajuba-Warts complex. Cell 158: 143-156.

Rauzi M, Lenne PF, Lecuit T. 2010. Planar polarized actomyosin contractile flows control epithelial junction remodelling. Nature 468: 1110-1114.

Rimm DL, Koslov ER, Kebriaei P, Cianci CD, Morrow JS. 1995. $\alpha_{1}(\mathrm{E})$-Catenin is an actin-binding and -bundling protein mediating the attachment of F-actin to the membrane adhesion complex. Proc Natl Acad Sci 92: 88138817.

Rosenblatt J, Raff MC, Cramer LP. 2001. An epithelial cell destined for apoptosis signals its neighbors to extrude it by an actin- and myosin-dependent mechanism. Curr Biol 11: 1847-1857. 
A.S. Yap et al.

Schlegelmilch K, Mohseni M, Kirak O, Pruszak J, Rodriguez JR, Zhou D, Kreger BT, Vasioukhin V, Avruch J, Brummelkamp TR, et al. 2011. Yap1 acts downstream of $\alpha$ catenin to control epidermal proliferation. Cell 144: 782-795.

Sebbagh M, Renvoize C, Hamelin J, Riche N, Bertoglio J, Breard J. 2001. Caspase-3-mediated cleavage of ROCK I induces MLC phosphorylation and apoptotic membrane blebbing. Nat Cell Biol 3: 346-352.

Silvis MR, Kreger BT, Lien WH, Klezovitch O, Rudakova GM, Camargo FD, Lantz DM, Seykora JT, Vasioukhin V. 2011. $\alpha$-Catenin is a tumor suppressor that controls cell accumulation by regulating the localization and activity of the transcriptional coactivator Yap1. Sci Signal 4: ra33.

Sinha B, Koster D, Ruez R, Gonnord P, Bastiani M, Abankwa D, Stan RV, Butler-Browne G, Vedie B, Johannes L, et al. 2011. Cells respond to mechanical stress by rapid disassembly of caveolae. Cell 144: 402-413.

Slattum G, McGee KM, Rosenblatt J. 2009. P115 RhoGEF and microtubules decide the direction apoptotic cells extrude from an epithelium. J Cell Biol 186: 693-702.

Smutny M, Cox HL, Leerberg JM, Kovacs EM, Conti MA, Ferguson C, Hamilton NA, Parton RG, Adelstein RS, Yap AS. 2010. Myosin II isoforms identify distinct functional modules that support integrity of the epithelial zonula adherens. Nat Cell Biol 12: 696-702.

Sugimura K, Lenne PF, Graner F. 2016. Measuring forces and stresses in situ in living tissues. Development 143: 186196.

Tamada M, Farrell DL, Zallen JA. 2012. Abl regulates planar polarized junctional dynamics through $\beta$-catenin tyrosine phosphorylation. Dev Cell 22: 309-319.
Tapon N, Harvey KF. 2012. The Hippo pathway-From top to bottom and everything in between. Semin Cell Dev Biol 23: $768-769$.

Trier SM, Davidson LA. 2011. Quantitative microscopy and imaging tools for the mechanical analysis of morphogenesis. Curr Opin Genet Dev 21: 664-670.

Vicente-Manzanares M, Ma X, Adelstein RS, Horwitz AR. 2009. Non-muscle myosin II takes centre stage in cell adhesion and migration. Nat Rev Mol Cell Biol 10: $778-790$.

Wen KK, Rubenstein PA, DeMali KA. 2009. Vinculin nucleates actin polymerization and modifies actin filament structure. J Biol Chem 284: 30463-30473.

Wu SK, Gomez GA, Michael M, Verma S, Cox HL, Lefevre JG, Parton RG, Hamilton NA, Neufeld Z, Yap AS. 2014. Cortical F-actin stabilization generates apical-lateral patterns of junctional contractility that integrate cells into epithelia. Nat Cell Biol 16: 167-178.

Yamada S, Pokutta S, Drees F, Weis WI, Nelson WJ. 2005 Deconstructing the cadherin-catenin-actin complex. Cell 123: 889-901.

Yao M, Qiu W, Liu R, Efremov AK, Cong P, Seddiki R, Payre M, Lim CT, Ladoux B, Mege RM, et al. 2014. Force-dependent conformational switch of $\alpha$-catenin controls vinculin binding. Nat Commun 5: 4525.

Yonemura S, Wada Y, Watanabe T, Nagafuchi A, Shibata M. 2010. $\alpha$-Catenin as a tension transducer that induces adherens junction development. Nat Cell Biol 12: $533-$ 542.

Yu H, Mouw JK, Weaver VM. 2011. Forcing form and function: Biomechanical regulation of tumor evolution. Trends Cell Biol 21: 47-56. 


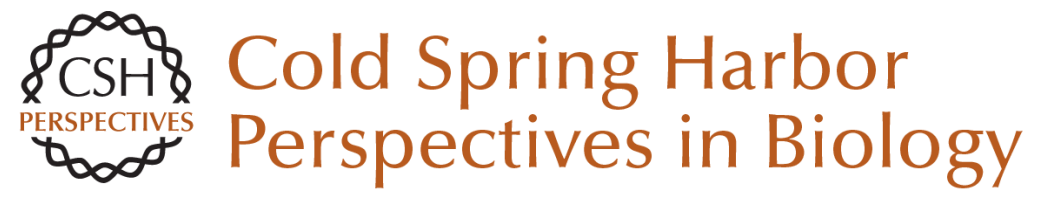

\title{
Mechanosensing and Mechanotransduction at Cell-Cell Junctions
}

\author{
Alpha S. Yap, Kinga Duszyc and Virgile Viasnoff
}

Cold Spring Harb Perspect Biol 2018; doi: 10.1101/cshperspect.a028761 originally published online August 4, 2017

\section{Subject Collection Cell-Cell Junctions}

Vascular Endothelial (VE)-Cadherin, Endothelial Adherens Junctions, and Vascular Disease Maria Grazia Lampugnani, Elisabetta Dejana and Costanza Giampietro

Adherens Junctions and Desmosomes Coordinate Mechanics and Signaling to Orchestrate Tissue Morphogenesis and Function: An Evolutionary Perspective Matthias Rübsam, Joshua A. Broussard, Sara A. Wickström, et al.

Cell-Cell Contact and Receptor Tyrosine Kinase Signaling Christine Chiasson-MacKenzie and Andrea I. McClatchey

Hold Me, but Not Too Tight--Endothelial Cell-Cell Junctions in Angiogenesis Anna Szymborska and Holger Gerhardt

\section{Connexins and Disease} Mario Delmar, Dale W. Laird, Christian C. Naus, et al.

\section{Cell Junctions in Hippo Signaling}

Ruchan Karaman and Georg Halder

Loss of E-Cadherin-Dependent Cell-Cell Adhesion and the Development and Progression of Cancer Heather C. Bruner and Patrick W.B. Derksen
Signaling by Small GTPases at Cell-Cell Junctions: Protein Interactions Building Control and Networks Vania Braga

Making Connections: Guidance Cues and Receptors at Nonneural Cell-Cell Junctions Ian V. Beamish, Lindsay Hinck and Timothy E. Kennedy

The Cadherin Superfamily in Neural Circuit Assembly James $D$. Jontes

Mechanosensing and Mechanotransduction at Cell-Cell Junctions Alpha S. Yap, Kinga Duszyc and Virgile Viasnoff

Beyond Cell-Cell Adhesion: Sensational

Cadherins for Hearing and Balance Avinash Jaiganesh, Yoshie Narui, Raul Araya-Secchi, et al.

Cell-Cell Junctions Organize Structural and Signaling Networks Miguel A. Garcia, W. James Nelson and Natalie Chavez

Cell Biology of Tight Junction Barrier Regulation and Mucosal Disease Aaron Buckley and Jerrold R. Turner

For additional articles in this collection, see http://cshperspectives.cshlp.org/cgi/collection/

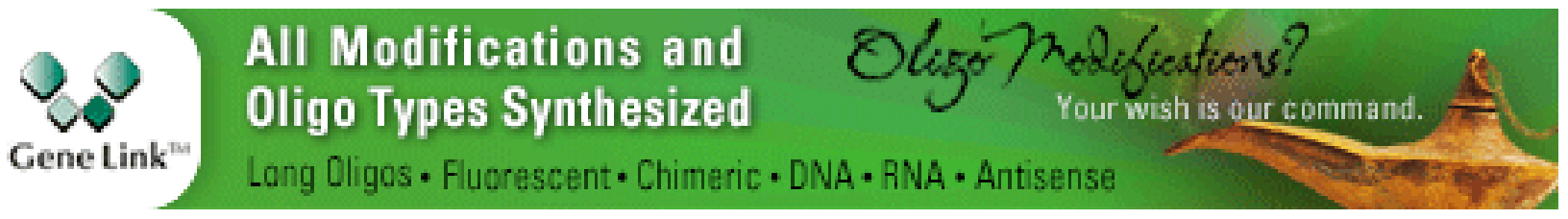


Desmosomes and Intermediate Filaments: Their Consequences for Tissue Mechanics Mechthild Hatzfeld, René Keil and Thomas M. Magin
Integration of Cadherin Adhesion and

Cytoskeleton at Adherens Junctions

René Marc Mège and Noboru Ishiyama

For additional articles in this collection, see http://cshperspectives.cshlp.org/cgi/collection/

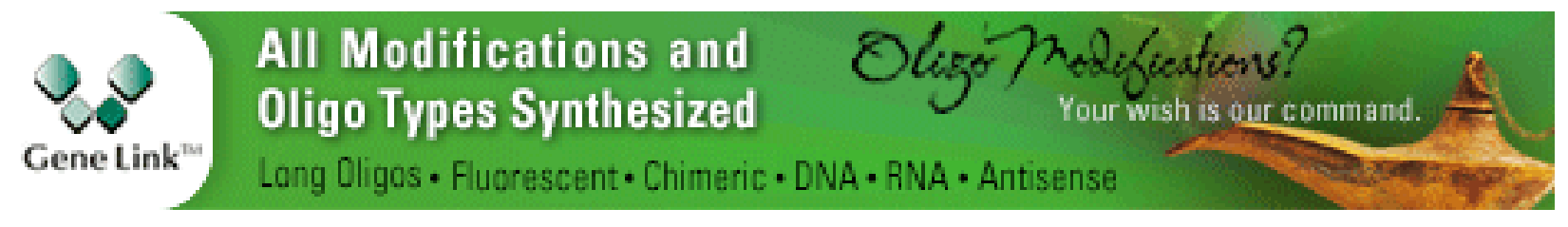

Copyright @ 2018 Cold Spring Harbor Laboratory Press; all rights reserved 